\title{
Behavioral Health Emergencies Encountered by Community Paramedics: Lessons from the Field and Opportunities for Skills Advancement
}

\author{
Bronwyn Keefe, MSW, MPH, PhD \\ Kelsi Carolan, MSW \\ Amy J. Wint, MSc \\ Matthew Goudreau, BS, NRP \\ W. Scott Cluett IIINRP \\ Lisa I. Iezzoni, MD, MSc
}

\begin{abstract}
Note: This statement is mandatory. Please provide.
\end{abstract}

\section{Introduction}

When an individual calls 911 because of a behavioral health emergency, paramedics may be the first health care professional on scene to address that crisis. Traditionally, the paramedic profession has been educated to respond to life-threatening medical emergencies. ${ }^{1}$ Paramedics are increasingly expected to respond to behavioral health crises but may not be adequately prepared to do so. ${ }^{1}$ There has been limited research regarding paramedics' experiences, attitudes, and perceptions about responding to behavioral health crises, with a particular dearth of literature on this subject from the USA. ${ }^{1-5}$ As the first point of contact, paramedics may influence the quality of care individuals experiencing behavioral health emergencies receive, rendering this gap in knowledge

Address correspondence to Bronwyn Keefe, MSW, MPH, PhD, School of Social Work, Boston University, 264 Bay State Road, Boston, MA 02215, USA.

Kelsi Carolan, MSW, School of Social Work, Boston University, Boston, MA, USA.

Amy J. Wint, MSc, Mongan Institute Health Policy Center, Massachusetts General Hospital, Boston, MA, USA.

Lisa I. Iezzoni, MD, MSc, Mongan Institute Health Policy Center, Massachusetts General Hospital, Boston, MA, USA. Matthew Goudreau, BS, NRP, EasCare Ambulance Company LLC, Dorchester, MA, USA.

W. Scott Cluett IIINRP, EasCare Ambulance Company LLC, Dorchester, MA, USA.

Lisa I. Iezzoni, MD, MSc, Department of Medicine, Harvard Medical School, Boston, MA, USA.

Publisher's Note Springer Nature remains neutral with regard to jurisdictional claims in published maps and institutional affiliations.

Journal of Behavioral Health Services \& Research, 2020. 365-376. (c) 2020 National Council for Behavioral Health. DOI 10.1007/s11414-020-09687-4 
concerning. ${ }^{1,4}$ Paramedics encounter a range of behavioral health crises, from individuals who have self-harmed, substance use, to older adults experiencing social isolation. ${ }^{6,7}$ This study aimed to answer the following questions: Do paramedics feel well prepared to respond to behavioral health crises? How do paramedics describe the challenges and facilitators involved in responding to these situations? What are the skills they use to respond to these situations? The present study examined paramedics' experiences and perceptions regarding behavioral health emergencies, aiming to address a significant gap in the literature on paramedics' ability to respond to the needs of individuals experiencing behavioral health crises.

\section{Background}

Limited research based in the UK, Europe, and Australia has examined the extent to which paramedics feel equipped by their training to respond to situations of a behavioral health nature, with a marked lack of USA-based research in this area. In a survey of members of the College of Paramedics (UK), 98\% of the 623 respondents endorsed the need for increased mental health education and training. ${ }^{2}$ Paramedic training curriculums in the UK have evolved to incorporate more of an emphasis on behavioral health, yet whether or not this behavioral health training is effective has not yet been investigated. ${ }^{1}$ Several Australian studies investigated paramedic perceptions of their mental health training and decision-making during mental health emergencies. ${ }^{5,8}$ Paramedic participants reported inadequate mental health training and indicated a need for increased education on mental illness, particularly regarding possible treatment options that are feasible as part of prehospital care. ${ }^{8}$ Paramedics described relying more on intuition than education when faced with challenging cases. ${ }^{5}$ In a mixed methods study, Australian paramedics reported the need for more education on how to adequately respond to older adults experiencing complex mental health crises including depression, social isolation, and food insecurity. ${ }^{7}$

Limited research has explored paramedic perceptions, attitudes, and experiences responding to behavioral health emergencies, but the existing research indicates that many emergency healthcare providers consider physical health emergencies more valuable or important than behavioral health emergencies. ${ }^{8-10}$ Studies indicate that paramedic students do not consider training in mental health issues as relevant to the profession, ${ }^{1}$ with an Australian study of paramedic undergraduate students indicating a lower level of regard for those with intellectual disabilities, substance abuse disorders, or acute mental illness compared to students studying in other health professions. ${ }^{11}$

A multi-site, qualitative study based in Paris, France and New York, New York examined how social and professional values influenced prehospital emergency workers' responses. ${ }^{9}$ The authors found that patients deemed to have lower social value were lower priority to emergency providers, with cases involving substance use or calls from lower socioeconomic areas, for instance, regarded as less legitimate uses of emergency services. ${ }^{9}$ Notably, situations that required more complex or heroic medical or surgical actions were also attributed higher value by emergency healthcare workers. ${ }^{9}$ Greater importance was attributed to medical (versus behavioral health) emergencies across additional studies, with paramedic participants implying that behavioral health calls were not valid emergencies, ${ }^{5}$ and with paramedic participants indicating that their role is mainly to transport — not to treat - individuals experiencing behavioral health crises. ${ }^{8}$ Additionally, paramedic participants perceived their services as often used inappropriately for behavioral health emergencies due to the limited availability of more appropriate behavioral health services. ${ }^{8}$

Related research regarding the perceptions of emergency department physicians and nurses in the USA also revealed a greater value placed on physical versus behavioral health emergencies, as well as perceptions that certain crises (such as self-harm) were less valid, or less worthy of emergency treatment. ${ }^{10}$ This is particularly concerning given that emergency department healthcare providers interact with a substantial number of persons seeking behavioral health emergency care: in 2015 , approximately $4.1 \%$ of visits to an emergency department were related to diagnosed 
mental health disorders (approximately 5,666,000 visits). ${ }^{12}$ The number of emergency department visits related to opioid overdose increased by $29.7 \%$ in the USA between July 2016 and September 2017. ${ }^{13}$ Emergency healthcare workers are treating a significant number of behavioral health emergencies, rendering improved understanding of healthcare providers' perceptions and attitudes towards this population crucial.

Existing research into the perceptions of experienced paramedic practitioners regarding behavioral health emergencies is limited. ${ }^{1,3,4}$ With the exception of a multi-site study based in New York, New York and Paris, France, ${ }^{9}$ a thorough review of the extant literature failed to unearth research on the perceptions and experiences of paramedics responding to behavioral health crises in the USA. This is a particularly significant gap, as differences in paramedic education, relevant behavioral health legislation, and culture may exist across countries. The present study used qualitative analyses of in-depth interviews of practicing paramedics to address this gap by examining paramedics' perceptions and experiences responding to behavioral health crises in the USA.

\section{Methods}

This study was part of a Patient-Centered Outcomes Research Institute (PCORI) contract funding an examination of the Acute Community Care Program (ACCP) at Commonwealth Care Alliance (CCA). The Acute Community Care Program is a state-funded community paramedicine intervention for the patients of CCA, in conjunction with EasCare Ambulance Company.

\section{Interview guide and recruitment}

The research team, which included experienced paramedics at EasCare Ambulance Company, worked together to create an open-ended guide to interview paramedics. The interview protocol consisted of six sections: (1) history of career as paramedic, (2) current skills and job experiences, (3) views of paramedic experience, (4) career goals, (5) final questions and wrap up, and (6) demographics. The research team revised the original guide after several pilot interviews and based on feedback from key informants, namely seasoned paramedic supervisors from the research team. Interviews with paramedics lasted approximately $1 \mathrm{~h}$ and were conducted over the phone by either the PI or Co-PI, who are experienced in qualitative research.

The research team intended to interview 25 paramedics who would be identified through two large ambulance companies in the Greater Boston area of Massachusetts. The director at these ambulance companies asked their paramedics on staff if they would be willing to be interviewed and, if they agreed, the director gave us their contact information. Six paramedics who had initially agreed could not be reached, and arrangements were made with one paramedic who did not follow through with the interview. In total, the researchers conducted telephone interviews with 23 paramedics, after receiving verbal informed consent to perform and record the interview. Participants were mailed a $\$ 50$ gift card to thank them for their time. Professional transcription service transcribed digital recordings verbatim, and the project manager reviewed all transcripts against the digital recording, making small corrections as needed.

\section{Data analysis}

Audiotapes of the sessions were analyzed by three research team members. A thematic analysis approach was utilized, starting with familiarization with the data, generating initial codes, searching for themes, reviewing themes, defining and naming themes, and producing a final analysis. ${ }^{14}$ The researchers worked both separately and collaboratively, working individually at each stage of the process and then coming together to compare results in order to ensure the 
validity of identified themes. Early readings of the transcript data allowed the researchers to develop preliminary ideas regarding potential themes. Each researcher then thoroughly analyzed the individual transcripts, generating initial codes and beginning to search for themes both within and across documents. During this stage, the researchers met frequently to compare and contrast the results of each individual's analysis, reviewing emerging themes and collaborating to refine themes into a coherent set. To asses inter-rater reliability, two un-read transcripts were reviewed by all team members and coded independently, followed by meetings as a research team to compare coding decisions on each transcript and reconcile any discrepancies. This rigorous process helped to ensure strong inter-rater reliability as we were able to gain consensus in our coding process for all other transcripts. Researchers individually arranged transcript data verbatim into groups of initial themes, and a master document was then created collaboratively, which outlined each finalized theme and included transcript excerpts embodying each theme.

Memo-writing was employed throughout the analytic process to document coding decisions. ${ }^{15}$ As a final check for validity, the two experienced paramedic supervisors from the research team reviewed the themes and subthemes and provided feedback about the findings. The paramedic supervisors confirmed that these themes were what they might have expected the data to reveal, further validating the findings.

\section{Results}

Table 1 shows demographic characteristics of the 23 participants. Results for this paper will focus on three themes identified in the data analysis. Namely, paramedics (1) report frequently working with patients who are having a behavioral health crisis, (2) report having inadequate behavioral health training, and (3) have many difficulties managing these patients, relying

\section{Table 1}

Paramedic characteristics $(N=23)$

\begin{tabular}{ll}
\hline Characteristic & Count \\
\hline Age, years & 40.9 mean (8) SD \\
Gender & 14 \\
Male & 9 \\
Female & \\
Race & 22 \\
White & 1 \\
Hispanic, Black & \\
Marital status & 18 \\
Married & 4 \\
Single & 1 \\
Divorced & 0 \\
Widowed & \\
Education & 4 \\
High school & 12 \\
Some college & 6 \\
Associate's Degree & 1 \\
Bachelor's degree & 18 \\
Average years in the field & \\
\hline
\end{tabular}


primarily on their professional experiences and/or strong interpersonal skills rather than explicit training to address patients' needs (see Table 2).

\section{Theme 1: paramedics work frequently with patients having a behavioral health crisis}

The paramedics interviewed described dealing with behavioral health emergencies as a routine part of the job, yet these were often some of the most difficult aspects of their job. Some of the more challenging situations arose around issues of self-harm, managing calls related to substance use, and maintaining personal safety. The overall toll this work can have on the paramedic's own behavioral health was also evident. Participants reported that the individuals they encounter often have limited support systems and many are socially isolated, which exacerbates behavioral health issues.

It's hard to know how to help someone that doesn't have anybody else to help them. Nobody else in the world, nobody I can call for that. They're by themselves, they're lonely. Not just the homeless population, but there's a lot of people at home, especially a lot of the elderly population. They just don't have anybody. That always breaks my heart.

The following quote describes an interaction a paramedic had with a woman experiencing debilitating grief after her son's death.

So there was one particular time and it was actually a mental health call. We went in and this woman was actually on the floor, huddled up in just a fetal position. She had lost her son in a rather aggressive battle with cancer. So she was very devastated and her husband was a three-time cancer survivor. I sat on the floor with her for about 15 minutes and I let her just tell me everything going on. And then once that was done, I don't even know where it came from, but I was like, "You know, your son would not want you to be here like this and you can't go backwards. What we can do is move forward and we can bring you to someone to go talk to and we can figure this out because, you know what, that should be your son's legacy, not this."

Table 2

Main themes and subthemes

Main themes

Paramedics work frequently with patients having a behavioral health crisis

Paramedics report inadequate behavioral health training

Experiential learning and strong interpersonal skills are critical tools

\section{Subthemes}

- They see a range of complex problems

- These are the most challenging to deal with

- This can have a personal toll on paramedics

- These interactions can be deeply meaningful

- Personal safety also has to be considered

- Training is either nonexistent or lacking in behavioral health

- Behavioral health training is needed to improve their competencies

- Other techniques are needed to effectively support patients

- Communication, empathy, and de-escalation are critical skills

- Skills are learned on the job as there is a lack of formal training

- Paramedics seek learning opportunities by self-study or asking questions when interacting with medical personnel at transferring facilities 
This paramedic drew on sophisticated communication skills and empathy, which was exemplified by several of the behavioral health calls paramedics described responding to, where no physical treatment was needed but the patient was clearly in crisis. Participants reported increasing numbers of calls involving opioid or other substance use, which they described as particularly challenging. This is articulated in the following quote:

\begin{abstract}
We actually do a lot because, for one, you've got the opioid epidemic that's going around. And some people would say, "Yeah, oh, it's just a drug. These people just want to do drugs and this and other." Sometimes there's a lot more. When you get into it, there's a lot more. The psychological issues that tend to turn them towards that and tend to keep them on it. Depression is a big one for alcohol and drugs. I mean, when you're depressed, you don't know what to do. Instead of going to a doctor, they take drugs or they drink. And we get taught how to deal with these people, that they're not crazy. That there's something going on. There's a real person here.
\end{abstract}

The above quote shows the paramedic's empathy towards the situation that person is in but this is not always the case. In particular, the following paramedic expresses how he feels he is not using the technical and medical skills he learned in his training and is merely responsible for transporting people to and from facilities who are impaired because of substance use concerns.

But a lot of the 911 work we're getting, it's the heroin overdose. It's the drunk. It was very routine, very-I don't want to say mundane, but I guess that's the best word I can come up with right now. Where, to me, getting a patient ... a cardiac arrest. They might've had a cath done. They have all these different meds running that you're titrating and trying to balance to keep the blood pressures up, and the heart rates going, and we got the vent going, and it's a little of everything you've learned in a call. I love working, using the knowledge that I've learned. Anyone could pick someone up who's drunk and bring him to the ER because they want to get a turkey sandwich that night because they know that hospital has turkey sandwiches. And doing things like that, that's very frustrating. You don't really feel like you're helping somebody. Where, these situations with these critical transfers, I actually just love them.

Personal safety when treating patients with mental health and substance use concerns is a key challenge, and varied approaches to this challenge were discussed, including use of medical or physical restraints or using techniques of de-escalation. The following quote exemplifies some of these challenges.

\footnotetext{
I had a 70-year-old lady flip out in the back of the truck, and it was really hard to try to restrain a 70-year-old lady. It was just kind of heartbreaking, but she was a danger. I mean, she clawed her way through some layers of skin. But it's trying to make sure that they're safe, while keeping yourself safe. It is scary when you're picking up somebody that's been known to have a little bit of a hard time. Sometimes, we'll call out for an extra crew, and we'll ask the extra crew to come out with us in the truck. So that way, they're kind of more outnumbered. So that way, it's easier to try to handle them.
}

In addition to potential physical risks, paramedics are often faced with situations that are emotionally challenging and can bring up many personal issues. The following quote demonstrates some of the strain a behavioral health emergency can have on a paramedic.

It was a suicide. A guy who was actually born two months before me. At that time, I had two daughters at home, he had two daughters. He had written an "I'm sorry" card to his oldest daughter and left it in his car. They had the same car seats and everything...That particular one, just because of how many things at that point in time, between the car seats, the cards to his kids, being so close in age, it really got me thinking about what could bring you to this point. That was probably the emotional hardest one.

The data illustrates that paramedics encounter patients who are struggling with behavioral health emergencies and describe these encounters as some of their most challenging and memorable onthe-job experiences.

\title{
Theme 2: paramedics report inadequate behavioral health training
}

When paramedics were asked about the amount or types of training they received in behavioral health, the majority expressed that it was lacking and not sufficient to equip them to do their job to full capacity. This is expressed by quite a few paramedics, as articulated below. 
They do have a section in it ... not that it doesn't cover half of what you need to, it doesn't cover a lot. It's a section. It's not a big portion of a section. It is a small portion actually.

Very little... they don't go into mental health a lot, and the extent of it is learning about behavioral emergencies that you may be called for, but that's about it. And then the rest of the training is how to not get injured in the situation like that and how not to make it worse by certain behaviors. But yet you're really coming at that acute point, but they don't really talk to you or teach you about more about diseases, they didn't go into in depth.

I feel like it's very minimal. They teach you to restrain your combative patient, and you try to deescalate your manic patients, and that's basically it.

Not really. And mental health was actually not part of that from what I can remember. And we actually deal with a lot of mental health patients.

Further, many paramedics expressed that additional behavioral health training would improve their competence and confidence in various treatment methods and approaches to support the person in crisis.

Maybe some more behavioral health and some addiction services [training], and social type of services. Not that we're going to be able to provide them, but maybe a better understanding of why "Joe's" on the street corner doing what he's doing. Some people that have been around a long time actually understand that. Sometimes the newer folks just don't have a handle on it, especially if they didn't grow up with that around them or if they don't understand it. Maybe some more work in addiction services.

\begin{abstract}
I would put more in there because we're coming across a lot more patients who have episodes where they're just completely not themselves, and they fight, and there's ways to go about talking to these people, where you don't end up in a physical altercation with these people, or having to physically restrain them with soft restraints, tying them to the stretcher. You know what I'm saying? But to teach enough ways to deal with them because I think it would be better to put more emphasis on that as well.
\end{abstract}

A common approach that came up in the encounters paramedics have with people who are experiencing a behavioral health crisis is the use of physical and medical restraints, interventions paramedics describe as critical to their safety.

I stand behind the fact that paramedics and any other pre-hospital ambulance type people need more training in behavioral help because really they don't give us any. No de-escalation techniques, they give you limited amount of leeway for how you can physically restrain patients. Even at the medic level, so now I can chemically sedate patients if they're full resistant to care or combative. But that's still a grey area because what you may say is resistant to care is different from what I may say is resistant to care. So it's a little difficult, and a lot of times we don't have the training so we don't really know what is a behavioral emergency. For me, it may be like okay, well I don't really like the story you're telling right now, so I'm going to sedate you. That's not the answer, right?

This paramedic describes the complexity of decision-making processes around safety in the context of behavioral health emergencies, indicating a lack of adequate training in this area. Participants expressed a lack of sufficient training in the areas of mental health and substance use even though they frequently encounter patients struggling in these areas.

\title{
Theme 3: experiential learning and strong interpersonal skills are critical tools
}

Participants reported that the experiences of interacting with patients having behavioral health crises has been central to their developing skills in this area, in lieu of adequate formal mental health training. Participants revealed a great capacity for empathy and strong interpersonal skills, which they rely on heavily when interacting with patients who are having behavioral health 
emergencies. Data demonstrated that there are certain characteristics that many paramedics share such as compassion, empathy, and strong interpersonal and communication skills. These skills are critical in many situations but perhaps are most essential when working with people who are dealing with mental health and substance use issues. The following quotes illustrate how paramedics see the person first and the disease or illness second - an important clinical skill that exemplifies the compassion many paramedics display.

I treat them with respect, listen, and don't be so judgmental. Everybody has a story and everybody has a problem and you just try to ... don't make them feel isolated.

When you take a psychiatric patient, you've got to step back and realize that sometimes they don't know what they're doing. So you try to step back and try to see it from their point of view.

Paramedics describe communication skills as both verbal and nonverbal and the importance of language and presence when helping those in the midst of a behavioral health crisis. In the following quote, the participant describes communication skills developed on the job, rather than formally taught.

You've got to be able to draw the information out from the person themselves. And it's a skill that they don't teach in school. They don't teach you how to talk to somebody. They don't teach you how to throw questions at somebody in a way that they'll answer and it'll lead to the next question. You set them up to answer in a way that gives you all that information. That's one thing the schools don't do. They don't teach you that part.

And my approach is, first, do no harm. If somebody's having a ... whether it's a true emotional crisis or, in fact, if it's somebody with known mental health problems, approaching them in a way that's nonthreatening. Stances, no arms crossed. Even my upright standing position, I would approach somebody that's had an emotional or psychological issue, on an angle. So if you were standing right in front of me and we're looking at each other eye to eye, in fact, my right foot would be back; it's on an angle. And that's for personal safety.

Another critical communication skill is de-escalation. Paramedics previously described learning some of that in their training, but most significantly, this is a skill that they learn on the job. The following quote discuss how this particular skill is a part of many of their interactions, whether medical or behavioral health.

For the most part, I've done pretty well being able to de-escalate. There only a few instances I can remember that we actually had people getting hurt. And so, yeah. I've had enough experience where I know when I can and can't deescalate something.

Paramedics often sought learning opportunities on their own or took advantage of interactions with other healthcare providers to increase their behavioral health knowledge. The following quotes support the importance of this method of learning and how paramedics have turned to selfteaching in this area.

It makes treating patients harder, because you don't really know. I've had to actually do the research and read about your schizophrenias, and your bipolars, and all those types of things, just to have some sort of understanding of what I'm dealing with when I do go for calls like that. But it wasn't really ... it gets mentioned in school. But it's not a topic that you go into depth on.

And most of the thing we learned for any social skills as far as how to help people in a social situation is definitely on the job. What I call compassionate care. It's all on the job. Nobody said, just sit down and teach you how to handle psychiatric out of control kind of thing. It's a little section in a big program.

So when somebody comes in with a substance abuse problem, alcohol problem, psychosis problem ... I mean, sometimes it's not even drugs or alcohol. Sometimes it's people just ... they're poor, or they don't have the means, or 
just hadn't been going to the doctor, and they're out of their medications, and it turns them psychotic. And then we bring them in, and now they're able to get to a doctor. So all the doctors constantly tell us what the newest techniques are for calming patients and sometimes a psychologist we'll run into ... they'll tell us, "Oh yeah. You see these symptoms or these signs on a patient, it's probably this. It's probably due to drugs, or it's due to a psychosis that's coming on because of the stuff they're taking." That's pretty much what we get.

On-the-job learning is a key technique in this type of work and is critical in developing new paramedics' skills, as articulated below.

My skill sets adapt over time. It always has. Every piece of information that I learn as I go, it gets added into my assessment for every single patient thereafter. So it's like a constant learning type of setting."

I'd say it was a combination of both the idea of learning from a lot of people who sort of were experts ... between everybody, you had somebody who could show you something based on a level of expertise that would take years and years to accomplish. So I learned a lot of tricks of the trade and things of that nature from people who were very open in giving me advice and telling me why they do things the way they do.

Despite the challenges behavioral health emergencies pose, many paramedics described these encounters as deeply meaningful, as they feel that they are impacting someone's life for the better.

Well, anytime you see someone who's injured or who's desperate and needs assistance, it feels good to help that person. Maybe they're at the lowest point in their life, maybe a drug addict or an alcoholic, and you talk to them, you try to address their needs, and get them on the right path. And you get a good feeling. Maybe you helped someone.

In summary, several key themes from paramedic interviews have been revealed throughout this paper, namely there are a variety of behavioral health emergencies paramedics respond to and there is a lack of adequate behavioral health training. Additionally, results revealed that paramedics compensate for this lack of behavioral health training by actively learning on the job to develop well-tuned communication skills, grounded in a deep desire to help others.

\section{Limitations}

This study was limited by both its geographical area and sample size. The research team interviewed paramedics in the Greater Boston area of Massachusetts from two different ambulance companies. Further studies should consider broadening the geographic reach to include suburban areas, which could help in the generalizability of these findings to other regions of the country.

\section{Implications for Behavioral Health}

The present study addresses a significant gap in the literature by examining the experiences, perceptions, and attitudes of paramedics responding to behavioral health crises in the USA. The data highlights the types of behavioral health crises paramedics frequently encounter, provides insight into paramedics' complex roles, and demonstrates the potential risks responding to these crises may impose on paramedics' personal safety and emotional well-being. Most notably, the data indicates a significant gap in paramedic training. Paramedics' training and certification explicitly emphasizes the skills and knowledge required for emergency, on scene care, which is predominately focused on trauma, resuscitation, and acute medical concerns, not behavioral health training. ${ }^{16,17}$ Additional training to appropriately respond to and work with individuals experiencing behavioral health emergencies will better serve the individuals in crisis, as well as paramedics and other emergency providers.

The finding that paramedics report limited behavioral health training and emphasize the need for additional training in this area is largely consistent with the extant literature. Studies 
of paramedics in the UK and Australia, as discussed above, reveal paramedics' reported lack of mental health training and desire for additional training in this area. ${ }^{2,5,8}$ Research on paramedic students in the UK indicated that such students did not consider mental health education as relevant to the profession. ${ }^{1}$ This discrepancy between the perceptions of students and the perceptions of the experienced paramedics that make up this study's sample may indicate a significant discrepancy between perceptions of versus the reality of paramedic work and may provide additional evidence of the current gaps in paramedic education in behavioral health. Future research should aim to investigate the perceptions of paramedic students in the USA to enhance understanding as to how to better prepare paramedic students to respond to behavioral health crises in the field.

Overall, additional research is needed to further understand the strengths and weaknesses of current behavioral health training for paramedics in the USA. Once certified, paramedics must undergo periodic recertification, which requires both exams and evidence of continuing education - this could be a key point of intervention for a robust behavioral health training. This data clearly demonstrates that the paramedic profession needs - and endorses the need for-additional behavioral health training: a review of paramedic education and training curriculums across the USA would aid understanding of how to improve upon current behavioral health education for paramedics. The effectiveness of fairly recently implemented behavioral health education for paramedics in the UK has not been investigated ${ }^{1,4}$-a similar gap appears to exist in the USA. Further research should evaluate existing behavioral health training for paramedics in order to better understand how current practices may be failing to adequately prepare paramedics in this area. Enhanced education and training on this subject has the potential to improve paramedics' ability to fully respond to the needs of patients in times of behavioral health crises. Although literature specifically focusing on prehospital providers is lacking, research on behavioral health training of emergency department providers indicates that such training has the potential to improve behavioral health care. ${ }^{18}$ Training in behavioral health for medical professionals has traditionally been thought of as secondary or less important, in the context of all that one needs to learn to be a competent medical practitioner, ${ }^{1}$ but as this study illustrates, interactions with patients who are having a behavioral health crisis are common and can be challenging. Improved behavioral health training, either in their time of certification or during times of recertification and continuing education, for paramedics may improve care for individuals experiencing behavioral health emergencies in the community and can be a key point of intervention.

Prior research indicated that paramedics and other emergency healthcare providers may place more social and medical value on physical care, particularly those that require heroic, fast-paced, and/or complex intervention. ${ }^{9}$ This value was also identifiable in this study's data, with one participant quoted above, for instance, describing his preference for responding to calls that allow him to utilize his technical skills, rather than acting as a "chauffeur." Existing research also indicates variable attitudes and perceptions among emergency healthcare workers regarding individuals with behavioral health challenges, with studies of paramedic students and emergency department providers endorsing lower regard for individuals presenting with behavioral health challenges such as substance use disorders or selfharm. ${ }^{10,11}$ However, the present study's findings illustrate the substantial compassion, care, and empathy that the majority of the paramedics in the sample demonstrate towards individuals experiencing mental health crises, with some participants describing some of these emergencies as among their most rewarding on-the-job experiences.

Paramedics are well-poised as first responders embedded in the community to serve as resources to behavioral health providers. Some paramedics in this study mentioned that they often serve as a source of referral to social services. Further research could work to develop policies and procedures to help strengthen the referral mechanism between paramedics and 
behavioral health providers. Again, training would strengthen this referral link as training can increase the paramedic's capacity to identify behavioral health concerns more effectively, therefore increasing their capacity to refer with greater confidence. Structured and standardized behavioral health training could serve as a key point of intervention, improving job satisfaction for paramedics by enabling them to more confidently manage increasingly complex behavioral health calls, and most importantly, better serving individuals with behavioral health disorders. Improved capacity for care at the prehospital stage of intervention could potentially reduce unnecessary emergency room transfers and/or medical interventions for people experiencing behavioral health emergencies, reducing potential harm and preventing additional distress to the patients served, and ultimately benefitting the health care system as a whole.

Funding information This work was funded through a Patient-Centered Outcomes Research Institute (PCORI) Award (IHS-1502-27,177).

\section{Compliance with ethical standards}

Disclaimer All statements in this report, including its findings and conclusions, are solely those of the authors and do not necessarily represent the views of the Patient-Centered Outcomes Research Institute (PCORI), its Board of Governors or Methodology Committee.

\section{References}

1. Brady, M. Pre-hospital psychosocial care: Changing attitudes. Journal of Paramedic Practice. 2012; 4(9): 516-525.

2. Berry, M. College of paramedics' evidence into mental health care and policing. Journal of Paramedic Practice. 2014; 6(10): 539-540.

3. Rees, N, Rapport, F, \& Snooks, H. Perceptions of paramedics and emergency staff about the care they provide to people who selfharm: Constructivist metasynthesis of the qualitative literature. Journal of Psychosomatic Research. 2015; 78(6): 529-535.

4. Rees, N, Rapport, F, Thomas, et al. Perceptions of paramedic and emergency care workers of those who self-harm: A systematic review of the quantitative literature. Journal of Psychosomatic Research. 2014; 77(6): 449-456.

5. Shaban, R. Paramedic clinical judgment of mental illness: Representations of official accounts. Journal of Emergency Primary Health Care. 2005; 3(4): 1-10.

6. Rees, N, Rapport, F, Snooks, H, et al. How do emergency ambulance paramedics view the care they provide to people who self-harm?: Ways and means. International Journal of Law and Psychiatry. 2017; 50: 61-67.

7. Ross, L, Jennings, P, \& Williams, B. Psychosocial support issues affecting older patients: A cross-sectional paramedic perspective. Inquiry: A Journal of Medical Care Organization, Provision and Financing. 2017; 54.

8. Roberts, L, \& Henderson, J. Paramedic perceptions of their role, education, training and working relationships when attending cases of mental illness. Journal of Emergency Primary Health Care. 2009; 7(3): 73.5KB.

9. Nurok, M., \& Henckes, N. Between professional values and the social valuation of patients: The fluctuating economy of pre-hospital emergency work. Social Science \& Medicine. 2009; 68(3): 504-510.

10. Hadfield, J, Brown, D, Pembroke, L et al. Analysis of accident and emergency doctors' responses to treating people who self-harm. Qualitative Health Research. 2009; 19(6): 755-765.

11. Williams, B, Boyle, M, \& Fielder, C. Empathetic attitudes of undergraduate paramedic and nursing students towards four medical conditions: A three-year longitudinal study. Nurse Education Today. 2015; 35(2): e18.

12. Rui, P. \& Kang, K. National hospital ambulatory medical care survey: 2015 emergency department summary tables. Atlanta, GA: Centers for Disease Control and Prevention: National Center for Health Statistics, 2015.

13. Vivolo-Kantor A, Seth P, Gladden R et al. Vital signs: Trends in emergency department visits for suspected opioid overdoses - United States, July 2016-September 2017. MMWR Morbidity Mortality Weekly Report 2018, 67: 279-285, Atlanta, GA: Centers for Disease Control and Prevention, 2018.

14. Braun, V \& Clarke, V. Using thematic analysis in psychology. Qualitative Research in Psychology. 2006; 3(2): 77-101.

15. Charmaz, K. Constructing Grounded Theory. 2nd ed., Introducing Qualitative Methods. London; Thousand Oaks, Calif.: Sage, 2014.

16. Raynovich W, Hums J, Stuhlmiller DF, et al. Critical care transportation by paramedics: A cross-sectional survey. Air Medical Journal. 2013;32(5):280-288. doi:https://doi.org/10.1016/j.amj.2013.05.008 
17. National Registry of Emergency Medical Technicians. Paramedic Certification. https://www.nremt.org/rwd/public/document/paramedic. Accessed April 25, 2018.

18. McCann, T, Clark, E, McConnachie, S. Accident and emergency nurses attitudes towards patients who self-harm. Accident and Emergency Nursing, 2006;14: 4-10.

Publisher's Note Springer Nature remains neutral with regard to jurisdictional claims in published maps and institutional affiliations. 